\title{
Ultrasensitive thermometer for atmospheric pressure operation based on a micromechanical resonator
}

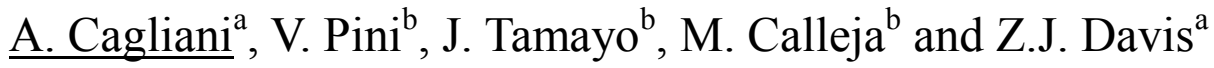 \\ ${ }^{a}$ DTU Nanotech, Institut for Mikro- og Nanoteknologi, Ørsteds Plads, Building 345Ø, 2800 Kgs. \\ Lyngby, Denmark \\ ${ }^{\mathrm{b}}$ Instituto de Microelectrónica de Madrid, Calle Isaac Newton 8, 28760 Tres Cantos, Madrid, Spain
}

Corresponding author: Alberto Cagliani, alberto.cagliani@nanotech.dtu.dk, +4545255759.

\begin{abstract}
For highly integrated systems for bio and chemical analysis a precise and integrated measurement of temperature is of fundamental importance. We have developed an ultrasensitive thermometer based on a micromechanical resonator for operation in air. The high quality factor and the strong temperature dependence of the resonance frequency of these bulk microresonators enable accurate temperature measurements. Here, we delineate the conditions to decouple the temperature effect on the resonance frequency, from the water adsorption/desorption on the resonator surface that happens when it is operated in air. This study enables high temperature resolution measurements, as well as the possibility of monitoring adsorption and desorption processes on the resonator surface with a resolution of 0.007 water molecules per $\mathrm{nm}^{2}$. These devices reach a temperature resolution of $3 \mathrm{mK}$ in air, which is one of best ever reported temperature resolution for resonator based thermometers.
\end{abstract}


Keywords: micromechanical resonator, temperature sensor, atmospheric pressure, mass sensitivity, humidity.

\section{Introduction}

Many material properties, such as the density, Young's modulus, refractive index, and resistivity depend on the temperature. This dependence has been harnessed to develop temperature sensors [1]. Among them, micromechanical based thermometer, play a considerable role both for research and commercial applications. As an example of a commercial resonator based thermometer, a resonant quartz thermometer is available from Hewlett-Packard with a resolution of $0.1 \mathrm{mK}$ [2]. However, quartz crystals are large devices with areas from 0.1 to several $\mathrm{mm}^{2}$ and cannot be scaled down in dimension and integrated with other silicon microdevices. In this respect miniaturized micromechanical resonators show promise for sensitive thermometers due to reduced size, high temperature sensitivity, low cost, and the easy integration with CMOS technology. Thanks to these advantages it has been suggested to use them as temperature sensors for active temperature compensation on-die of oscillating circuits [3-4].

In recent years, several microfabricated resonator based thermometers have been proposed [3-7]. In these devices, the resonance frequency and quality factor depend on the temperature via several mechanisms: the young's modulus dependence [7], the dependence of the Q-factor on the thermoelastic losses [3-4] and the temperature dependence of the internal stress of the resonator [5-6]. These microthermometers are based on flexural mode resonators reaching resolution of $8 \mathrm{mK}$ [7] and $2 \mathrm{mK}$ [3] only when operated in vacuum and these resolutions are comparable with the performance 
of CMOS based temperature sensors [8][9]. Unfortunately these devices are not suitable to be operated in air because the Q-factor in air is highly reduced by one to three orders of magnitude due to the viscous damping and this hampers drastically the temperature resolution [10-12]. Moreover, the degradation of the vacuum encapsulation causes unwanted drifts in the performance of the mechanical resonators [13].

Directly integrated thermometers that can work in air with very high resolution are demanding in many applications such as: monitoring cell activity via calorimetry [145], investigation of DNA-water interaction [16], photothermal analysis of airborne nanoparticles [16] infrared detection [17-20]. Moreover, in microfabricated gas analysis systems the control of the temperature of several elements is fundamental: the temperature of the gas chromatographic columns, the temperature of the gas, the temperature of the gas detectors during sensing and regeneration [21-22]. Despite the large range of possible applications, in recent years, only few examples of resonator based thermometers that operate at atmospheric pressure or in air have been proposed $[18-20,23]$. Verd et al. reached a resolution of $19 \mu \mathrm{K}$ in air at a constant relative humidity of $30 \%$, but considering that the device is also very sensitive to humidity a strong coupling between these effects should be expected [23][24]. To the best of our knowledge only one work deals with the performance of a micromechanical resonator in air at different relative humidities [25]. In order to achieve extreme temperature sensitivity in air, we have drastically shrunk the resonator dimensions, increasing the resonant frequency and entering the regime where the frequency shift induced by the 
water molecules adsorption/desorption process from the resonator surface is comparable with the temperature induced frequency shift.

We have designed a thermometer based on a micromechanical resonator that vibrates in an extensional mode with high quality factor even at atmospheric pressure and we have studied the resonance frequency dependence both on the temperature and the relative humidity establishing the conditions to uncouple the two physical parameters. The device reaches a temperature resolution of $3 \mathrm{mK}$ at $5 \%$ relative humidity $(\mathrm{RH})$ as well as being able to monitor the desorption of water molecule with a resolution of 0.007 water molecules per $\mathrm{nm}^{2}$ from the resonator surface (at constant temperature), thanks to its extreme distributed mass sensitivity of $14.8 \mathrm{kHz} \mu \mathrm{m}^{2} / \mathrm{fg}$ (to be compared to values in Table 1 in reference 22). Considering that one monolayer corresponds to $\sim 1$ water molecules per $\mathrm{nm}^{2}$ the device is able to precisely monitor adsorption and desorption processes happening on the surface [16].

\section{Materials and Methods}

The devices were placed in a custom made environmental chamber, where the relative humidity $(\mathrm{RH})$ and the temperature of the resonator are controlled during the entire experiment. The chip containing several devices was placed on top of a Peltier element, while the temperature was monitored by a high quality thermistor fixed against the chip using thermal glue from Artic Silver ${ }^{\mathrm{TM}}$. The RH in the environmental chamber was controlled by mixing dry nitrogen $\left(\mathrm{N}_{2}\right)$ and wet nitrogen $\left(\right.$ dry $\mathrm{N}_{2}$ bubbling through a glass bottle filled with fresh Milli-Q water) using precision valves. The humidity was changed within a working range from $1 \%$ up to $95 \%$ with a 
resolution of $0.1 \%$, while the temperature of the resonator was controlled using a closed loop electronics with a resolution of $0.05^{\circ} \mathrm{C}$. The calibration curve of the thermistor used is shown in Figure 1S.

The experiments reported in Figure 3a are performed sequentially. First the humidity is set below $2 \%$ for several hours. Afterwards it is set to $5 \%$ and, keeping constant this parameter, the temperature is raised up to $7^{\circ} \mathrm{C}$ with steps of $1{ }^{\circ} \mathrm{C}$, while the resonance frequency is monitored. After that, the temperature is set back to the lab temperature and the relative humidity is raised to the next value, then the temperature sweep is repeated.

\section{Results and Discussion}

\subsection{Longitudinal Mechanical Bulk Acoustic Resonator (LBAR)}

The longitudinal bulk acoustic micromechanical resonator (LBAR) used in this work consists of a polysilicon bar of $60 \mu \mathrm{m}$ in length, which is electrostatically driven into resonance. The microfabrication process used to fabricate the LBAR is based on the process used in [26], where two photolithographic+dry etching steps are used. The first step defines the body of the resonator with the LBAR shape into a heavily doped polysilicon-on-insulator layer, whereas the second defines the electrodes around (into a second polysilicon layer). Finally, the resonator is suspended and separated from the electrodes by etching away the $\mathrm{SiO}_{2}$ underneath the resonator and in the resonator-electrodes gap. Figure 1a shows a scanning electron microscopy (SEM) image of the resonator. The device is operated in a longitudinal extensional mode, as shown in Figure 1b. The device is composed by two cantilevers, which are moving 
along the length in an antiphase motion [27]. This mode is actuated electrostatically, applying a constant and a time varying potential to the electrodes. The resulting motional current is detected by capacitive readout, thanks to the two electrodes in close proximity to the resonator ends (see Figure 1c).

Table 1. LBAR data.

\begin{tabular}{|l|c|l|l|c|}
\hline & $\mathrm{Q}$ & Slope $@ f_{0}\left[{ }^{\circ} / \mathrm{Hz}\right]$ & $\sigma_{\text {phase }}\left[^{0}\right]$ & Frequency noise $[\mathrm{Hz}]$ \\
\hline Dry air & $\sim 2000$ & 0.0075 & 0.02 & 2.7 \\
\hline $0.25 \mathrm{mBar}$ & $\sim 13000$ & 0.04 & 0.016 & 0.4 \\
\hline
\end{tabular}

In Figure 2 the characterization of one of the devices is shown. The resonance frequency of the LBAR used in this work is $49.13 \mathrm{MHz}$ (in vacuum) and the Q-factor is about 13000 in vacuum $(0.25 \mathrm{mBar})$ and 2000 in air environment (room temperature and $\mathrm{RH}<2 \%$ ). In Figure $2 \mathrm{~b}$ and Figure $2 \mathrm{~d}$ the resonating signal after the mathematical compensation of the parallel capacitance are shown. The mathematical compensation technique consists in subtracting from the resonant signal as measured (Figure $2 \mathrm{a}$ and Figure 2c) the capacitive signal measured with $\mathrm{V}_{\mathrm{dc}}=0 \mathrm{~V}$ (i.e. no motional current) [26][28]. The frequency noise has been estimated for the two conditions by multiplying the inverse of the slope of the phase signal at resonance by the standard deviation of the phase noise [26]. The data for one device are reported in Table 1 . The resulting frequency noises averaged over three devices are $3 \mathrm{~Hz}$ for atmospheric conditions (relative humidity $5 \%$ ) and $0.3 \mathrm{~Hz}$ in vacuum with the acquisition bandwidth of the analyzer set to $30 \mathrm{~Hz}$ in both cases. 
The resonance frequency of an LBAR can be approximated by

$f_{0}=1 / 4 \mathrm{~L} \sqrt{E /\left(1+2 \mathrm{~A}_{e x} / w L\right) \rho}$

, where $\mathrm{L}$ is the length of one cantilever, $\rho$ is the density of polysilicon $\left(2320 \mathrm{~kg} / \mathrm{m}^{3}\right)$,

$\mathrm{A}_{\mathrm{ex}}$ the extra areas at the end, $\mathrm{w}$ is the resonator width and $\mathrm{E}$ is the polysilicon

Young's modulus (152 GPa). Under vacuum conditions, the resonance frequency varies linearly with the temperature because of the thermal expansion and Young's modulus variation. The temperature sensitivity is given by

$S_{\alpha, E}=\left.\frac{d f_{0}}{d T}\right|_{T=T_{0}}=\frac{\partial f_{0}}{\partial E} \frac{\partial E}{\partial T}+\frac{\partial f_{0}}{\partial L} \frac{\partial L}{\partial T}+\frac{\partial f_{0}}{\partial \rho} \frac{\partial \rho}{\partial T}=\frac{f_{0}}{2}\left(C_{E}+\alpha\right)$

, where $C_{E}$ is the temperature coefficient of the polysilicon Young's modulus, $\alpha$ is thermal expansion coefficient and $\mathrm{T}_{0}$ is the ambient temperature. However, in humid environment the adsorption and desorption of water must be taken into account and equation (2) is no longer valid.

The temperature dependence of the LBAR resonance frequency has been first studied in vacuum $\left(10^{-5} \mathrm{mBar}\right)$. We measured the resonance frequency within a range of 12 ${ }^{\circ} \mathrm{C}$ (see Supplementary Information, Figure 2S) and from the slope of the linear fitting we estimated a temperature sensitivity of $-1293 \mathrm{~Hz} /{ }^{\circ} \mathrm{C}\left(-25.1 \mathrm{ppm} /{ }^{\circ} \mathrm{C}\right)$. The contribution of the Young's modulus can be extracted by using equation (2) and it results to be $-47.6 \mathrm{ppm} / \mathrm{K}$ (if $\alpha=2.6 \mathrm{ppm} / \mathrm{K}$ ). Only two references are available for this material parameter. In reference [29] the value is $-74.5 \mathrm{ppm} / \mathrm{K}$, while in reference [30] is $-30 \mathrm{ppm} / \mathrm{K}$. The same material parameter for crystal silicon is $-60 \mathrm{ppm} / \mathrm{K}$ [31]. Therefore, our result falls within the range of possible values. Using the frequency noise previously found, our LBAR resonator based thermometer has a 
temperature resolution of $0.3 \mathrm{mK}$ (with an acquisition bandwith of $30 \mathrm{~Hz}$ ) under vacuum conditions. This resolution is one of the best resolutions found in literature for a microresonator based thermometer operated in vacuum [3-7].

\subsection{LBAR temperature and relative humidity dependence}

As mentioned in the introduction, a micromechanical resonator is subjected to changes in the relative humidity when operated in air, and consequently in the amount of water that condenses on its surface varies. As shown in this section, the resonance frequency of our LBAR resonators varies not only due to a change in temperature but also owing to the change of condensed water mass. This is due to the change in the total mass of the device that leads to a change in the resonance frequency following the well-known inverse relationship mass-resonance frequency (see equation 1). In Figure 3 we report the relative frequency shift obtained by changing the temperature at a fixed $\mathrm{RH}$ (red circles, $\mathrm{RH}=5 \%$ ) and by changing the humidity at a constant temperature (blue squares, $\mathrm{T}=27^{\circ} \mathrm{C}$ ); the error bars are calculated as average of 3 different resonators data. The adsorption isotherm data of the LBAR can be partially explained by the BET theory [32], which can be used to describe the adsorption of water molecules on the resonator's surface

$\frac{1}{N\left(\frac{1}{R H}-1\right)}=\frac{1}{c N_{m}}+\frac{c-1}{c N_{m}} R H$

where $\mathrm{RH}$ is the relative humidity defined as $\varphi / \varphi_{0}$ being $\varphi$ and $\varphi_{0}$ the partial pressure of water vapor and the saturation pressure respectively, $N$ is total number of water molecules adsorbed, $N_{m}$ is number of water molecules adsorbed in 1 monolayer 
and $\mathrm{c}$ is the BET constant defined as $\left.c=\exp \left[\left(E_{a}-E_{l}\right) / R T\right)\right]$, where $E_{a}$ is the adsorption energy, $E_{l}$ the liquefaction heat, $\mathrm{R}$ the gas constant and $\mathrm{T}$ the temperature. In our case the number of molecules adsorbed is proportional to the frequency shift that they induce, so equation (3) can be rewritten as $\frac{1}{\Delta f\left(\frac{1}{R H}-1\right)}=\frac{1}{c \Delta f_{m}}+\frac{c-1}{c \Delta f_{m}} R H$

where $\Delta f$ is the relative frequency shift with respect the resonance frequency at $1 \%$ $\mathrm{RH}, \Delta f_{m}$ is frequency shift that corresponds to the adsorption of one monolayer of water molecules. Fitting the BET isotherm to the adsorption data at $27^{\circ} \mathrm{C}$, a BET constant of 3.98 and a monolayer shift of $1.4 \mathrm{kHz}$ are calculated and the heat of adsorption of water on our native silicon dioxide surface is estimated to be 43.8 $\mathrm{kJ} / \mathrm{mol}$ (see supplementary information for the fitting details). This value is in good agreement with the literature [33].

As can been seen in Figure 3 the BET isotherm does not correlate with the adsorption data after $35 \%-40 \%$; this is due to the high surface tension of the water film on the resonator surface which is no taken into account in the simple BET model used. The deviation starts when more than one water layer is completed $(\mathrm{RH}>35-40 \%)$, due to in plane interactions between water molecules $[34,35]$. The heat of adsorption can be correctly calculated because is extracted only from the adsorption data between $5 \%$ and $35 \%$ relative humidity.

The comparison between the temperature and relative humidity dependence curves indicates that the frequency shifts induced by a temperature change or a humidity change have a comparable magnitude. This means that the resonance frequency shift 
of a LBAR in an environment where temperature and humidity are changing at the same time is then a superimposition of the two effects. In order to decouple the problem we have measured the temperature sensitivity at different values of relative humidity. This will permit us to measure the only the temperature effect (knowing the right temperature sensitivity) when the relative humidity is measured independently and constant in time.

\subsection{LBAR temperature sensitivity as a function of RH}

The inset in Figure 4a presents the resonance frequency vs. temperature curves at two different relative humidity values for the same LBAR microresonator. From the data we can conclude that, even if the two curves are linear (as confirmed by the $\mathrm{R}^{2}$ values), they have different slopes $\left(-19 \pm 0.5 \mathrm{ppm} /{ }^{\circ} \mathrm{C}\right.$ at $5 \% \mathrm{RH}$ and $-13.7 \pm 0.2 \mathrm{ppm} /{ }^{\circ} \mathrm{C}$ at $50 \% \mathrm{RH})$.

The resonator behavior is explained by the combination of two effects: i) increasing the temperature the resonance frequency decreases, because the Young's modulus of polysilicon also decreases and the resonator expands (like in vacuum); ii) increasing the temperature the resonance frequency increases, because some of the condensed water on the resonator surface evaporates. This means that the evaporation from the surface is partially counterbalancing the effect from the Young's modulus and the expansion, making the resonance frequency of the device less sensitive to the temperature. This concept is expressed as

$S_{T}(R H)=S_{E, \alpha}+S_{d}(R H)$ 
where $S_{T}\left[\mathrm{~Hz} /{ }^{\circ} \mathrm{C}\right]$ is the overall temperature sensitivity of the device, that is the superposition of a negative contribution $S_{E, \alpha}$ coming from the Young's modulus and the thermal expansion and a positive contribution $S_{d}$ coming from the desorption. Since $S_{d}$ depends on the relative humidity, the overall temperature response $S_{T}$ will vary with the humidity as well. In Figure $4 a$ the slopes of the linear temperature to frequency responses are reported for different humidities. The error bars are calculated using the data recorded for three different LBAR devices. The slope value varies from $-1015 \pm 41 \mathrm{~Hz} /{ }^{\circ} \mathrm{C}\left(-20 \pm 0.8 \mathrm{ppm} /{ }^{\circ} \mathrm{C}\right)$ at $5 \%$ to $-477 \mathrm{~Hz} /{ }^{\circ} \mathrm{C}\left(-9.7 \pm 2 \mathrm{ppm} /{ }^{\circ} \mathrm{C}\right)$ at $65 \%$. The temperature slopes are evaluated up to $65 \% \mathrm{RH}$ with linear fits with a $\mathrm{R}^{2}$ of at least 0.995 . The $\mathrm{R}^{2}$ is lower than 0.995 for relative humidity values higher than $65 \%$ in a 5 degrees range. Within the temperature range explored not measurable hysteresis has been detected at any relative humidity value. Considering the frequency noise of $3 \mathrm{~Hz}$ (see section 2.1), the temperature noise of the device in atmospheric conditions (at $5 \%$ relative humidity) is $3 \mathrm{mK}$. All the resonance frequency values obtained are measured when the equilibrium is reached for any set of conditions.

In Figure 4a the solid line is calculated using the temperature dependence of the fitted BET isotherm found in the previous section $\left(\mathrm{c}=3.98\right.$ and $\left.\Delta f_{0}=1.4 \mathrm{kHz}\right)$. The BET isotherm permits to calculate the frequency shift due to amount of adsorbed water mass that is desorbed per degree when the temperature is risen. This contribution is then subtracted to $S_{E, \alpha}$ at each relative humidity to calculate the theoretical $S_{T}$ line (see Supplementary Information for more details about BET adsorption isotherm 
temperature dependence). The experimental slope values are reproduced correctly up to $40 \% \mathrm{RH}$, the subsequent deviation is due to the fact the BET is not able to correctly calculate the adsorbed water mass when more than one water layer is present (see Figure 2) [34,35]. Figure 3b shows a 2D surface contour plot that illustrates the dual functionality of the LBAR resonators by reporting the relative frequency shift as a function of the temperature and the relative humidity, showing clearly how the devices are more sensitive to temperature at low relative humidity and are more sensitive to relative humidity at low temperature. In order to operate the device as thermometer the humidity has to be independently measured and vice versa, when operated as hygrometer the temperature has to be independently measured. To calculate the relative frequency shifts in Figure $3 b$ the data from Figure $3 a$ and Figure 2 are used and the zero is set for $27^{\circ} \mathrm{C}$ and $1 \%$ RH. The black line in Figure $3 \mathrm{~b}$ represent the line of zero frequency shift and corresponds to the curve where $S_{E, \alpha}$ and desorption effects $S_{d}$ are equal. This last aspect is not detrimental because we could take advantage of this compensation effect for the design of highly stable NEMSdevices. When the RH is higher than $65 \%$ the temperature response deviates from linearity, due to the non-linearity in the $S_{d}$ term and a slope value is not representative anymore (see supplementary information).

\section{$\underline{2.4 \text { Water mass calculation }}$}

The ability of monitoring the resonance frequency both with respect to temperature and relative humidity opens up the possibility of precisely quantifying the water mass that is exchanged at the resonator surface during the experiments shown in Figure 4. 
In order to calculate this quantity we need to evaluate the distributed mass sensitivity $S_{m}\left[\mathrm{kHz} \mu \mathrm{m}^{2} / \mathrm{fg}\right]$ of the resonator, defined as the frequency shift produced by a change of mass on resonator's surface

$S_{m}=\Delta f A_{r} / \Delta m$

, where $\Delta f$ is the frequency shift induced by an uniform distributed added mass layer $\Delta m$ and $A_{r}$ is the total area of the LBAR resonator. When the $S_{m}$ of the device is known, the variation of water mass, that is adsorbed on or desorbed from the device surface can be calculated by multiplying the inverse of $\mathrm{S}_{m}$ by the frequency shift measured [26].

We used a FEM simulation to estimate the $S_{m}$ value for the first extensional mode and resulted to be $14.8 \mathrm{kHz} \mu \mathrm{m}^{2} / \mathrm{fg}$. The validity of value has been confirmed theoretically and experimentally (see supplementary information). Thanks to this extreme mass sensitivity is possible to quantify the amount of water adsorbed or desorbed from the resonator surface when the temperature is changed at constant relative humidity (see Figure 5). The RH dependence of the desorbed water per unit degree $D_{w}(\mathrm{RH})$ has been obtained with the equation

$D_{w}(R H)=\frac{S_{T}(R H)-S_{E, \alpha}}{S_{m}}$

where $S_{E, \alpha}=S_{T}(R H=0)$ is the device's temperature sensitivity at dry conditions. As it is shown in Figure 5, the desorbed water from the resonator's surface quickly grows with the relative humidity and it reaches around one water molecule per $\mathrm{nm}^{2}$ and Celsius degree at $\mathrm{RH}=60 \%$. These measurements demonstrate the huge potential of our LBAR devices in terms of precisely monitoring the adsorption and desorption 
of molecules from the resonator surface. This is possible thanks to a mass resolution of $200 \mathrm{zg} / \mu \mathrm{m}^{2}$, calculated by multiplying the frequency noise found in section 2.1 with the inverse of $S_{m}$. This resolution corresponds to 0.007 water molecules per $\mathrm{nm}^{2}$ and moreover, it should be considered that a monolayer of water molecules on crystalline $\mathrm{SiO}_{2}$ has a density of $\sim 1$ molecules per $\mathrm{nm}^{2}$ [33], which is $\sim 140$ times our mass resolution.

\section{Conclusions}

In conclusion, we have demonstrated the use of a LBAR resonator as an ultrasensitive thermometer in air environment. After some preliminary measurements that reveal the extreme temperature resolution of $300 \mu \mathrm{K}$ in vacuum conditions, we performed the characterization of the device's performance also in humid environment by changing both the temperature and the relative humidity. Remarkably, thanks to the high quality factor of the LBAR resonators, we achieved the temperature resolution of $3 \mathrm{mK}$ in air environment. Moreover, we have demonstrated the possibility to decouple the temperature and the relative humidity dependence of the LBAR's resonance frequency, by measuring the temperature dependence at different relative humidities. Thanks to the extreme mass sensitivity, the water desorption has been characterized and a mass resolution of $200 \mathrm{zg} / \mu \mathrm{m}^{2}$ $\left(0.007\right.$ water molecules per $\left.\mathrm{nm}^{2}\right)$ has been estimated. These results pave the way of a miniaturized ultrasensitive thermometer able to work in humid environment and hold great potential for studying gas adsorption and desorption processes. 


\section{Acknowledgments}

We acknowledge financial support from the Spanish Science Ministry (MINECO) through projects MAT2012-36197; and from European Research Council through Starting Grant NANOFORCELLS (ERC-StG-2011-278860). The authors are very thankful to the personnel at DTU-Danchip for the help during the microfabrication and to Tom Larsen for fruitful discussion and technical help.

\section{Bibliography}

[1] P R N Childs, J R Greenwood, and C A Long. Review of temperature measurement. Review of Scientic Instruments, 71(8), 2000.

[2] Hewlett Packard model 2804A Quartz Thermometer

[3] M. A. Hopcroft, B. Kim, S. Chandorkar, R. Melamud, M. Agarwal, C. M. Jha, G. Bahl, J. Salvia, H. Mehta, H. K. Lee, R. N. Candler, and T. W. Kenny. Using the temperature dependence of resonator quality factor as a thermometer. Applied Physics Letters, 91(1):013505, 2007.

[4] Bongsang Kim, Matthew a. Hopcroft, Rob N. Candler, Chandra Mohan Jha, Manu Agarwal, Renata Melamud, Saurabh a. Chandorkar, Gary Yama, and Thomas W. Kenny. Temperature Dependence of Quality Factor in MEMS Resonators. Journal of Microelectromechanical Systems, 17(3):755-766, June 2008.

[5] T. Larsen, S. Schmid, L. Gronberg, a. O. Niskanen, J. Hassel, S. Dohn, and A. Boisen. Ultrasensitive string-based temperature sensors. Applied Physics Letters, 98(12):121901, 2011.

[6] Pandey, A. K., Gottlieb, O., Shtempluck, O., Buks, E. (2010). Performance of an AuPd micromechanical resonator as a temperature sensor. Applied Physics Letters, 96(20), 203105.

[7] C. M. Jha, G. Bahl, R. Melamud, S. A. Chandorkar, M. A. Hopcroft, B. Kim, M. Agarwal, J. Salvia, H. Mehta, and T. W. Kenny. High resolution microresonator-based digital temperature sensor. Applied Physics Letters, 91(7):074101, 2007.

[8] Makinwa, K. a. a. (2010). Smart temperature sensors in standard CMOS. Procedia Engineering, 5, 930-939.

[9] Udrea, F., Santra, S., \& Gardner, J. W. (2008). CMOS temperature sensors - concepts, state-ofthe-art and prospects. In 2008 International Semiconductor Conference (Vol. 44, pp. 31-40). IEEE. 
[10] Blom, F. R. (1992). Dependence of the quality factor of micromachined silicon beam resonators on pressure and geometry. Journal of Vacuum Science \& Technology B: Microelectronics and Nanometer Structures, 10(1), 19

[11] R A Buser, N. F. D. R. (1990). Very High Q-Factor Resonator in Monocrystalline Silicon. Sensors and Actuators A: Physical, 23, 323-327

[12] Yasumura, K. Y.; Stowe, T. D.; Chow, E. M.; Pfafman, T.; Kenny, T. W.; Stipe, B. C.; Rugar, D. Quality factors in micron- and submicron-thick cantilevers. J. Microelectromechanical Syst. 2000, 9, 117-125.

[13] Kaajakari, V.; Kiihamäki, J.; Oja, A.; Pietikäinen, S.; Kokkala, V.; Kuisma, H. Stability of wafer level vacuum encapsulated single-crystal silicon resonators. Sensors Actuators A Phys. 2006, 130-131, 42-47.

[14] Ren K., Kao P., Pisani M. B., and Tadigadapa S. Monitoring biochemical reactions using Y-cut quartz thermal sensors. Analyst, 136 (14), 2904-11, 2011.

[15] Lee, W., Fon, W., Axelrod, B. W., \& Roukes, M. L. (2009). High-sensitivity microfluidic calorimeters for biological and chemical applications. Proceedings of the national Academy of Sciences USA, 106(36).

[16] A. Cagliani, P. Kosaka, J. Tamayo and Z. J. Davis. Monitoring the hydration of DNA self-assembled monolayers using an extensional nanomechanical resonator. Lab on a chip, 12(11), 2069-73, 2012.

[17] Larsen, T.; Schmid, S.; Villanueva, L. G.; Boisen, A. Photothermal analysis of individual nanoparticulate samples using micromechanical resonators. ACS Nano 2013, 7, 6188-93.

[18] Tsow F., and Tao N. Microfabricated tuning fork temperature and infrared sensor. Applied Physics Letters, 90(17), 174102, 2007.

[19] Wang Z., Member S., Qiu X., and Member S. (2010). Film Bulk Acoustic-wave Resonator ( FBAR ) Based Infrared Sensor. Response, (d), 824-827.

[20] Zhang, X. C.; Myers, E. B.; Sader, J. E.; Roukes, M. L. Nanomechanical torsional resonators for frequency-shift infrared thermal sensing. Nano Lett. 2013, 13, 1528-34.

[21] Agah, M.; Potkay, J. A.; Member, S.; Lambertus, G.; Sacks, R.; Wise, K. D. High-Performance Temperature-Programmed Microfabricated Gas Chromatography Columns. $J$. Microelectromechanical Syst. 2005, 14, 1039-1050.

[22] Lee, A. P.; Reedy, B. J. Temperature modulation in semiconductor gas sensing. Sensors Actuators B. Chem. 1999, 60, 35-42.

[23] Verd, J., Sansa, M., Uranga, A., Perez-Murano, F., Segura, J., \& Barniol, N. (2011). Metal microelectromechanical oscillator exhibiting ultra-high water vapor resolution. Lab on a Chip, 11(16), 2670-2. doi:10.1039/c1lc20103d

[24] Verd, J., Sansa, M., Uranga, A., Pey, C., Abadal, G., Perez-Murano, F., \& Barniol, N. (2009). Monolithic CMOS-MEMS oscillators with micro-degree temperature resolution in air conditions. In TRANSDUCERS 2009 - 2009 International Solid-State Sensors, Actuators and Microsystems Conference (pp. 2429-2432). IEEE. doi:10.1109/SENSOR.2009.5285435 
[25] Hosseinian, E.; Theillet, P.-O.; Pierron, O. N. Temperature and humidity effects on the quality factor of a silicon lateral rotary micro-resonator in atmospheric air. Sensors Actuators A Phys. 2013, 189, 380-389.

[26] Cagliani A., and Davis Z. J. (2011). Ultrasensitive bulk disk microresonatorbased sensor for distributed mass sensing. Journal of Micromechanics and Microengineering 21, 045016.

[27] J. H. Hales, J. Teva, a. Boisen, and Z. J. Davis. Longitudinal bulk acoustic mass sensor. Applied Physics Letters, 95(3):033506, 2009.

[28] Lee, J. E.-Y., Zhu, Y., and Seshia, A. (2008). A bulk acoustic mode single-crystal silicon microresonator with a high-quality factor. Journal of Micromechanics and Microengineering, 18(6), 064001. doi:10.1088/0960-1317/18/6/064001

[29] Rutigliano C. R. Guckel H., Burns D. W., Tilmans A. C., Deroo D. W. Mechanical properties of negrained polysilicon the repeatability issue. Solid state sensor and Actuator Workshop, pages 96-99, 1988 .

[30] K. Wang, A.C. Wong, and T.-C. Nguyen. VHF free-free beam high-Q micromechanical resonators. Journal of Microelectromechanical Systems, 9(3):347-360, 2000.

[31] Matthew A. Hopcroft, William D. Nix, and Thomas W. Kenny. What is the Young's Modulus of Silicon? Journal of Microelectromechanical Systems, 19(2):229-238, April 2010.

[32] Brunauer, S.; Emmett, P. H.; Teller, E. Adsorption of Gases in Multimolecular Layers. J. Am. Chem. Soc. 1938, 60, 309-319.

[33] Mizushima S. Determination of the amount of gas adsorption on $\mathrm{SiO}_{2} / \mathrm{Si}$ (100) surfaces to realize precise mass measurement. Metrology, 41:137-144, 2004.

[34] McMillan, W. G.; Teller, E. The Role of Surface Tension in Multilayer Gas Adsorption. $J$. Chem. Phys. 1951, 19, 25.

[35] McMillan, W. G.; Teller, E. The Assumptions of the B.E.T. Theory. J. Phys. Chem. 1951, 55, $17-20$.

Dr. Alberto Cagliani, Lecco, Italy, 1983. He obtained his Ph.D. from the Danish Technical University in 2011. His studies were focused on high frequency micromechanical resonators for ultrasensitive mass detection and temperature measurements. He is currently working for the Center of Excellence for Nanostructured Graphene at DTU on innovative techniques for nanostructuring graphene.

Valerio Pini obtained the bachelor and the master degrees in physics from University of Florence in 2005 and 2008 respectively. He received the master degree in nanotechnology from Universidad Autonoma de Madrid in 2010. He is currently a Ph.D. student at the Institute of Microelectronics of Madrid. His research activities are focused on the experimental and theoretical development of nano-mechanical devices for bio-sensing applications. 
Dr. Javier Tamayo, Madrid, Spain, 1970. PhD in Physics by the University Complutense of Madrid in 1998. Since 2001 he has been a Research Staff member at the IMM/CNM/CSIC and he is leading the Bionanomechanics Group, devoted to the application of micro- nanomechanical structures for biological interfacing. He has 6 licensed patents and has published 77 articles in indexed journals, which have received more than 3600 citations. He has leaded more than ten national and international projects along the last five years. Javier Tamayo is scientific promoter and founder of the spin-off company Mecwins S.L.

Dr. Montserrat Calleja Ourense (Spain) 1973. Senior Researcher at the Microelectronics Institute of Madrid-CSIC (Spain), where she has been head of Dept. of Devices, Sensors and Biosensors from 2008-2012. Since 2004 she has focused her research in nanomechanical sensing, developing new instrumentation and technologies for the application of nanomechanics to Biology. She has coauthored more than 60 publications, leaded more than 10 research projects and filled 8 patents. In 2008 she launched thecompany Mecwins S.L. fot the commercialization of nanomechanical sensors for clinical diagnosis. Her present research interests are in the study of the mechanical properties of living cells.

Dr. Zachary James Davis was born in July 1974 in Tulsa, Oklahoma, USA. Zachary received his $\mathrm{PhD}$ in Electrical Engineering in 2003 at the Technical University of Denmark within the field of nanomechanical mass sensors. In 2004-2005 Zachary worked at Epson GmbH and he started his own research group in 2006 within the field of dynamic NEMS as Associate Professor, focusing on field of nanomechanical resonators. In 2011 Zachary joined the Danish Technological Institute, where he is coordinating several projects related to cleanroom processing of surface acoustic wave components, sensor technologies and is also working within micro fuel cells. 

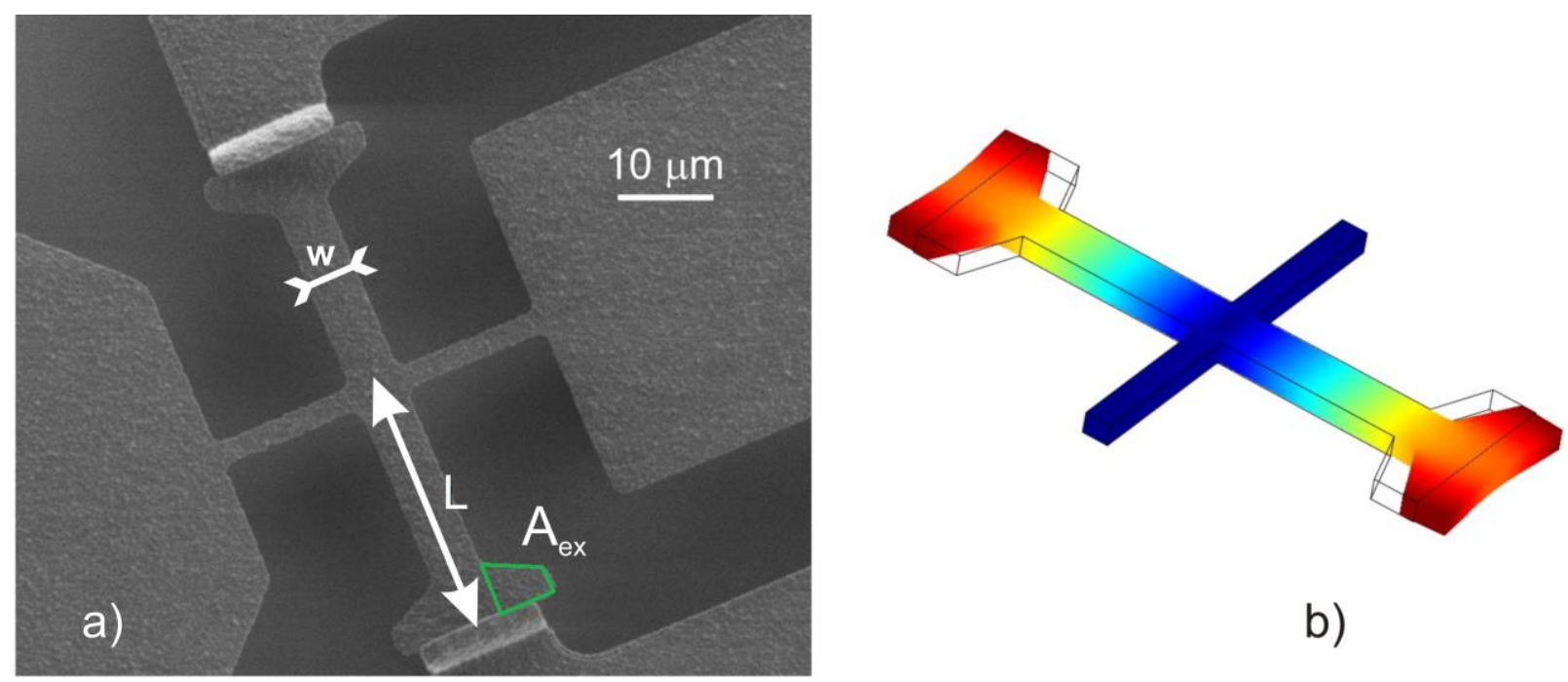

b)

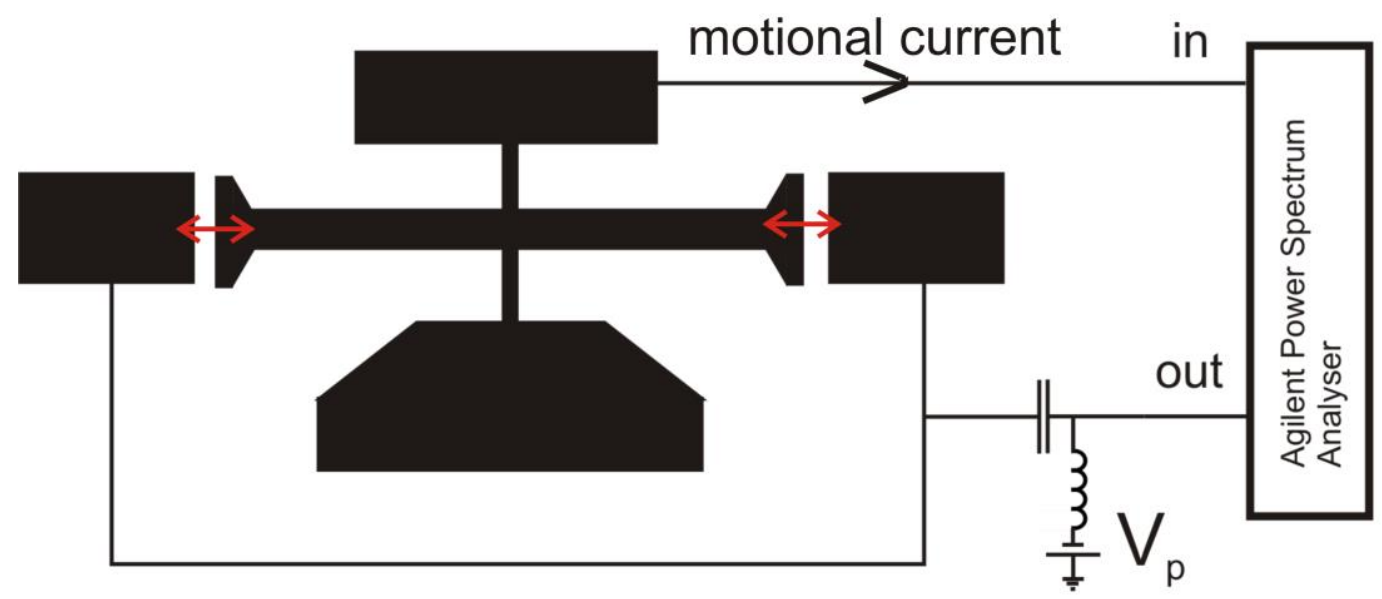

c)

Figure 1. LBAR Resonator and electrical scheme used.

(a) SEM micrograph of the LBAR resonator: the width $w=6 \mu \mathrm{m}$, the length $L=30 \mu \mathrm{m}$, the thickness is $2.5 \mu \mathrm{m}$ and the extra area $A_{e x}$ are indicated. The gap between the electrodes and the resonator body is $100 \pm 5 \mathrm{~nm}$. (b) Finite element simulation of the first longitudinal mode shape of vibration for a LBAR (dark blue corresponds to no movement, dark red to maximum movement). (c) Actuation and readout scheme. A power spectrum analyzer has been connected to the resonator as well as to a DC source. 

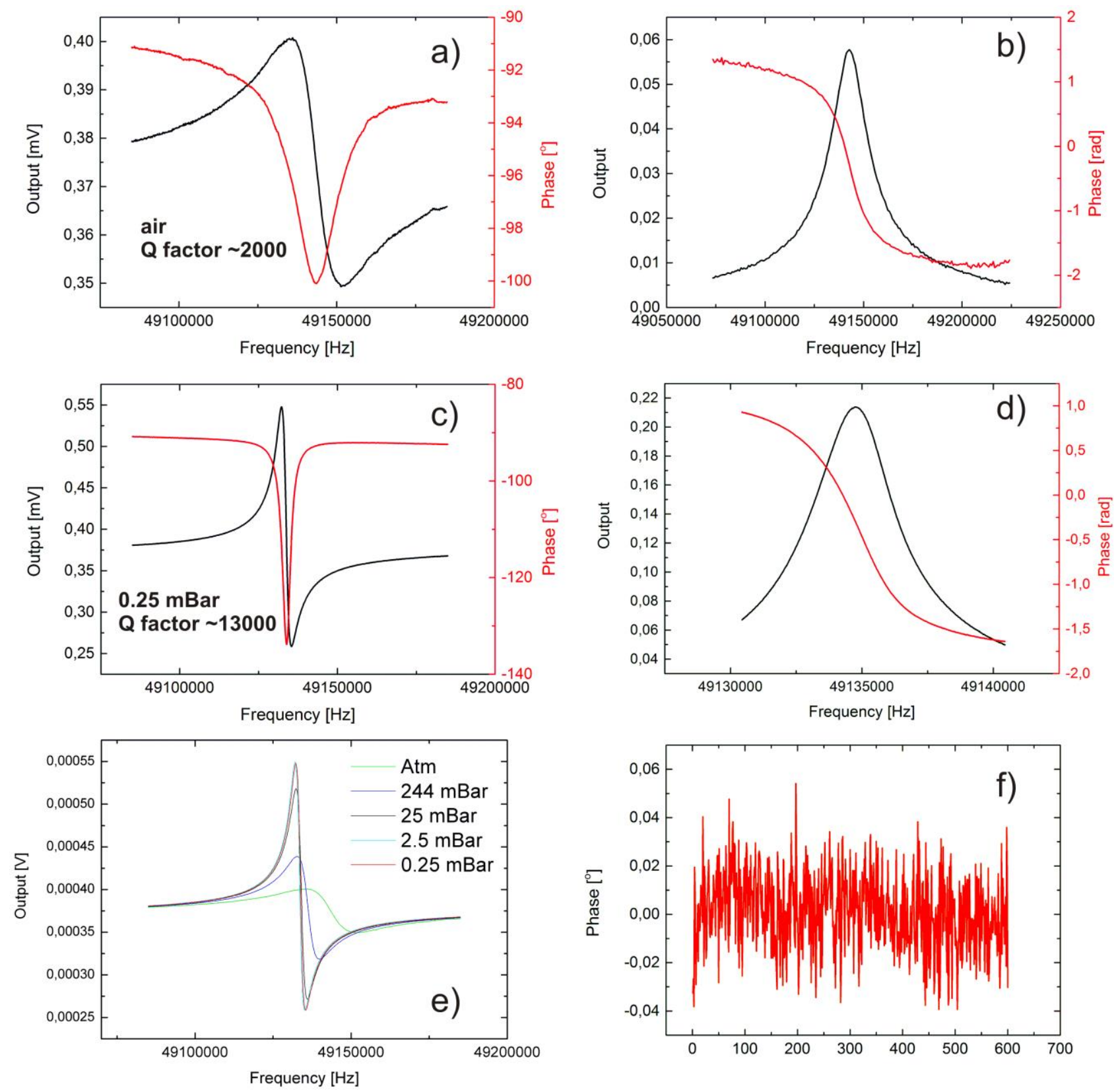

Figure 2. (a) Frequency response in air $\left(V_{d c}=3 V\right.$ and $\left.V_{a c}=0.36 V\right)$. (b) Compensated frequency response in air. (c) Frequency response in vacuum. (c) Compensated frequency response in vacuum. (d) Evolution of the frequency response from atmospheric pressure to $0.25 \mathrm{mBar}$. (f) Zero frequency span acquisition of the phase at $49.135 \mathrm{MHz}$ in vacuum with the device connected with $V_{d c}=3 \mathrm{~V}, V_{a c}=0.36 \mathrm{~V}$ and an acquisition bandwidth of $30 \mathrm{~Hz}$. The data have been centered around zero. 


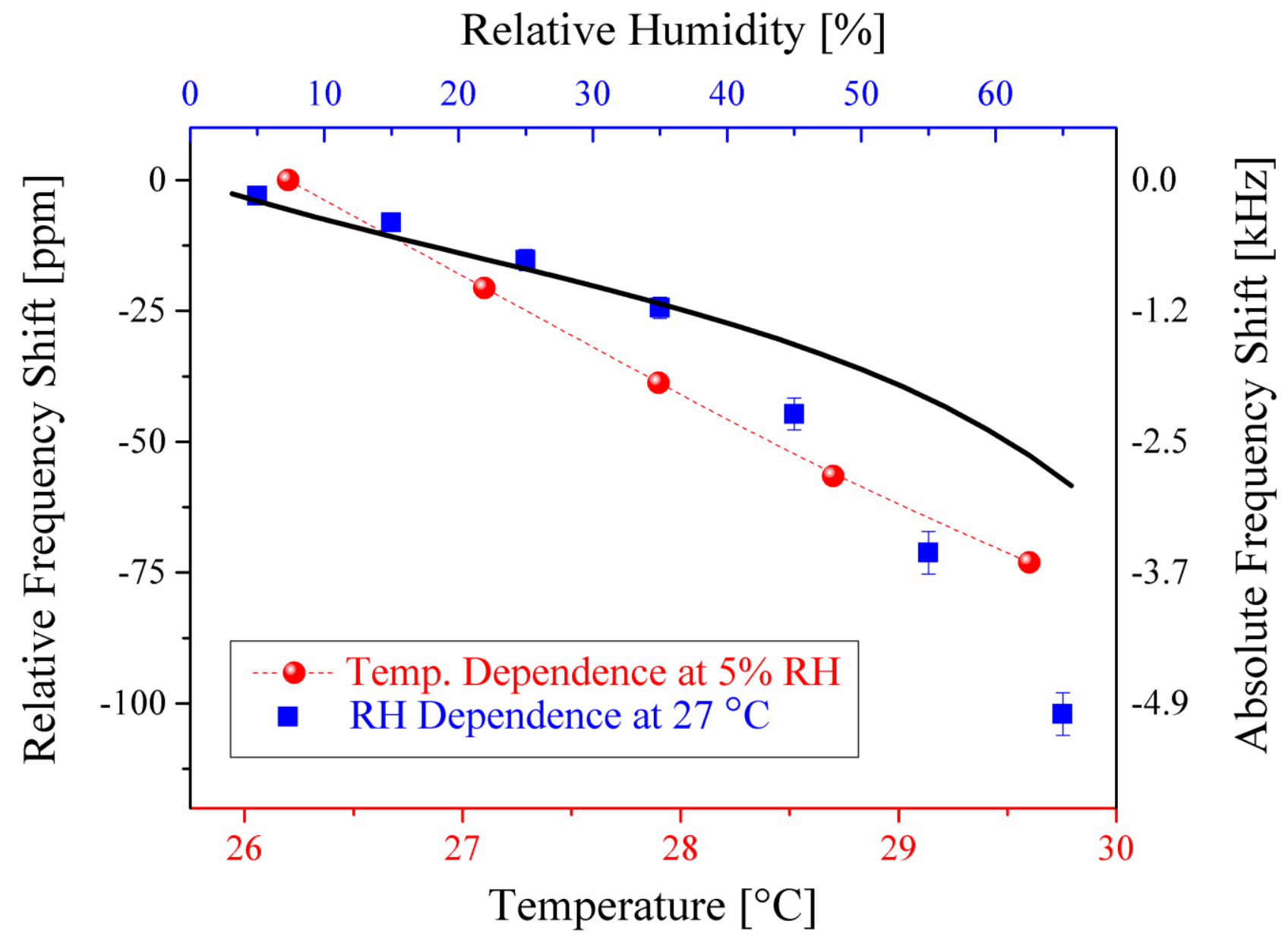

Figure 3. Temperature and relative humidty dependence.

Red Circles: Temperature dependence of the relative frequency shift recorded at $R H=5 \%$. The reference frequency is given by the resonance frequency measured at the temperature $T=26.2{ }^{\circ} \mathrm{C}$. The red dashed line is a guide for the eyes. Blue squares: Adsorption isotherm of the LBAR acquired at $27^{\circ} \mathrm{C}$. Black line represents the fitted $B E T$ isotherm (BET constant $c=3.98, \Delta f_{m}=1.4 \mathrm{kHz}$ ). 

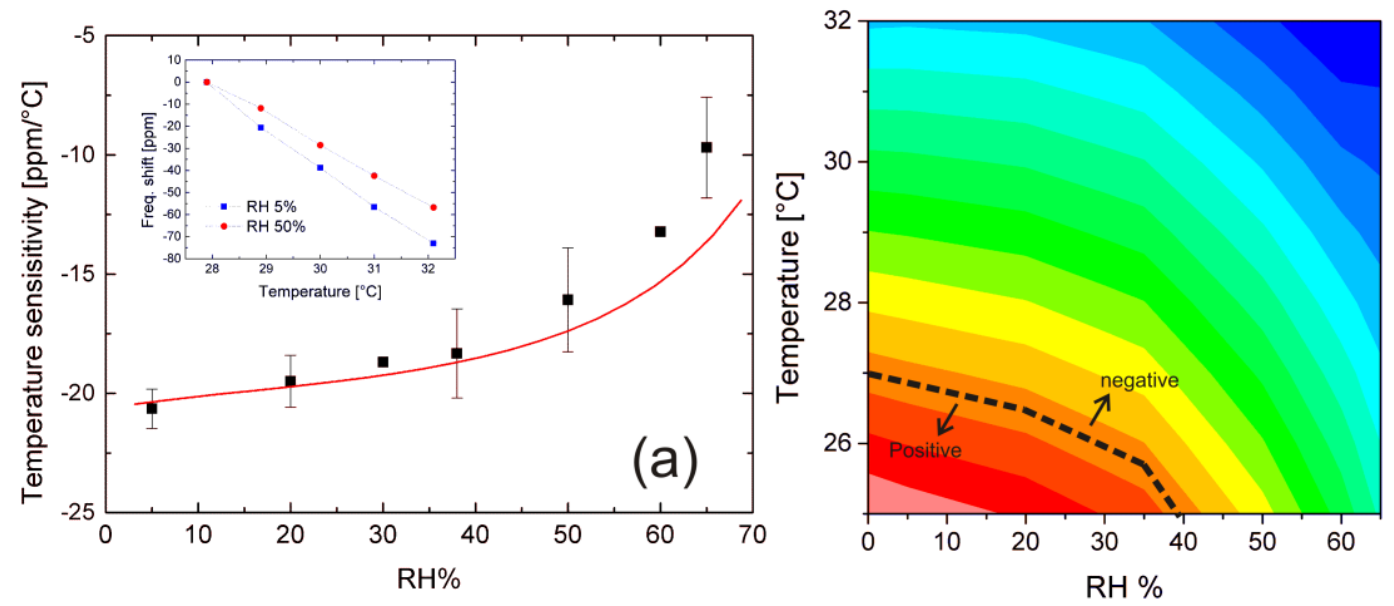

Relative Freq. shift [ppm]

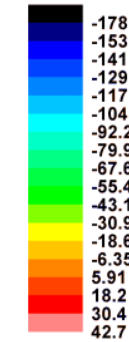

(b)

Figure 4. Variation of the temperature sensitivity with the relative humidity.

(a) (Insert) Temperature response at two different relative humidities for the same LBAR resonator. The slope is $-19 \pm 0.5 \mathrm{ppm} /{ }^{\circ} \mathrm{C}$ at $\mathrm{RH}=5 \%$, while is $-13.7 \pm 0.2 \mathrm{ppm} /{ }^{\circ} \mathrm{C}$ at $R H=50 \%$ ( $R^{2}$ is 0.996 and 0.998 respectively). The error bars are smaller than the data markers. (Main) Temperature sensitivity at different relative humidity values. The reported data are averaged over 3 resonators. For $\mathrm{RH} 30 \%$ and $60 \%$ only one device has been measured. The red line represents the prediction of the slope values using the temperature dependence of the fitted BET isotherm. (b) 2D plot of the dependence of the resonator frequency on temperature and on relative humidity. The zero is set at $T=27^{\circ} \mathrm{C}$ and $\mathrm{RH}=1 \% \mathrm{RH}$. 


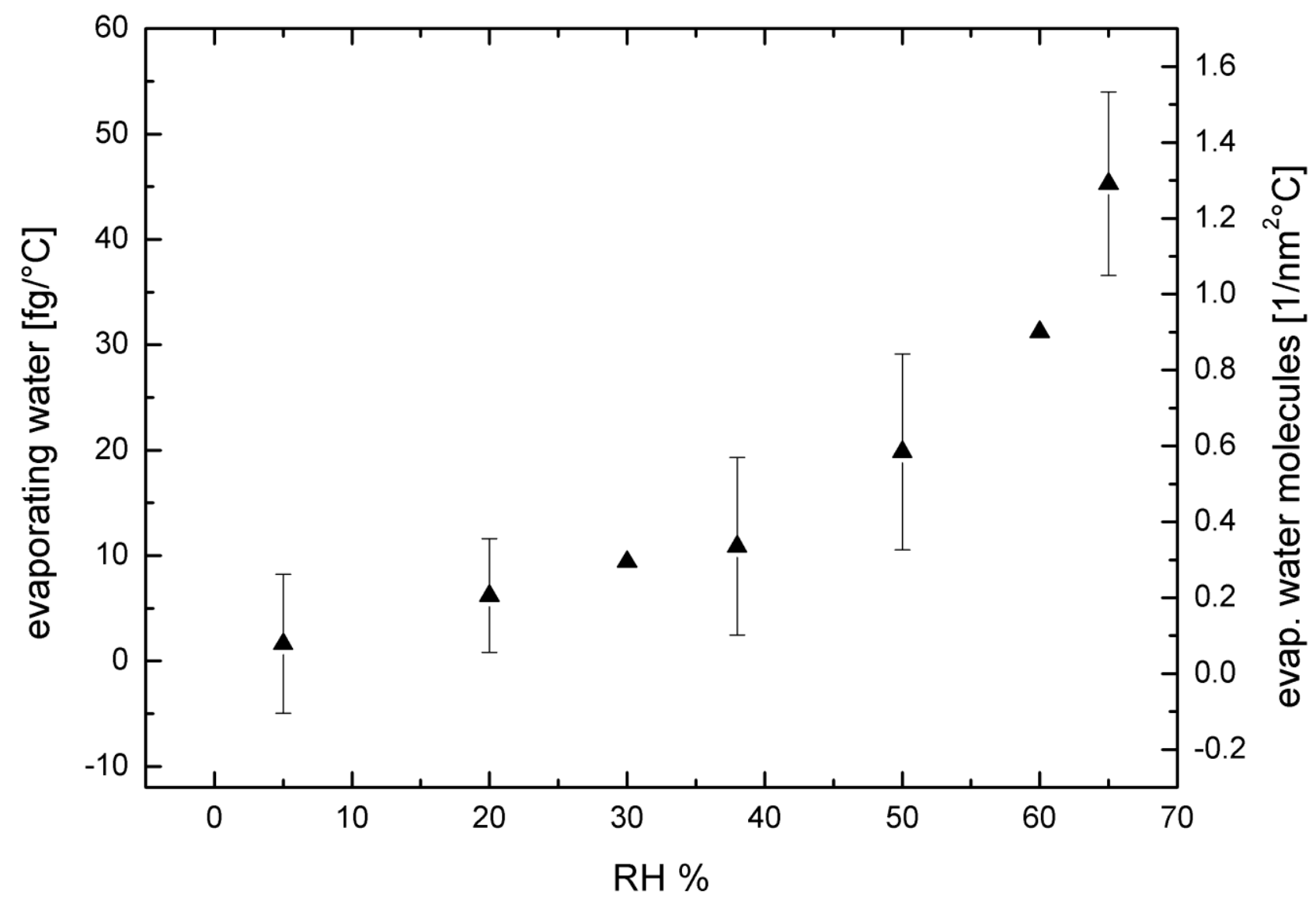

Figure 5. Water molecule desorption at different relative humidity values.

Relative humidity dependence of the water mass desorbed per $\mathrm{nm}^{2}$ and Celsius degree (left axis) and of the number of water molecules per $\mathrm{nm}^{2}$ and Celsius degree (right axis). The error bars are calculated using the data of three resonators. 